\title{
SILICON MONOXIDE IN SUPERNOVA SN1987A
}

\author{
Craig H. Smith, David K. Aitken \\ Dept. of Physics, University College ADFA, Campbell, ACT, 2601, Australia \\ Patrick F. Roche \\ Dept. of Astrophysics, University of Oxford, Oxford, OX1 3RH, U.K.
}

\begin{abstract}
The $8.1 \mu \mathrm{m} \Delta v=1$ emission band of silicon monoxide detected in $\mathrm{SN}$ $1987 \mathrm{~A}$ is modelled. Near day 500 the SiO mass is $4 \pm 210^{-6} \mathrm{M}_{\odot}$ and the excitation temperature is $\sim 1500 \mathrm{~K}$. The mass of $\mathrm{SiO}$ is about 10 percent of the mass of dust inferred from the mid infrared emission near day 600 , while the temperature is close to the condensation temperature of silicate grains. The $\mathrm{SiO}$ molecules may have been precursors to dust grain formation.
\end{abstract}

\section{Introduction}

- Silicon Monoxide was clearly present in the infra-red spectrum of SN 1987a from about day 160 to day 517, but could have been present at both earlier and later times.

- Spectra taken with the UCL spectrometer on the Anglo-Australian Telescope on days 465 and 517 show the $\mathrm{SiO}$ emission clearly between 8 and $9.5 \mu \mathrm{m}$, while the data between 9.5 and $13 \mu \mathrm{m}$ allow the level and slope of the continuum emission to be established.

- We have extracted the SiO band emission by adopting a continuum of slope $\mathrm{F}_{\lambda} \propto \lambda^{-3.7}$ on day 465 and $\lambda^{-3.5}$ on day 517 (Fig 1 ).
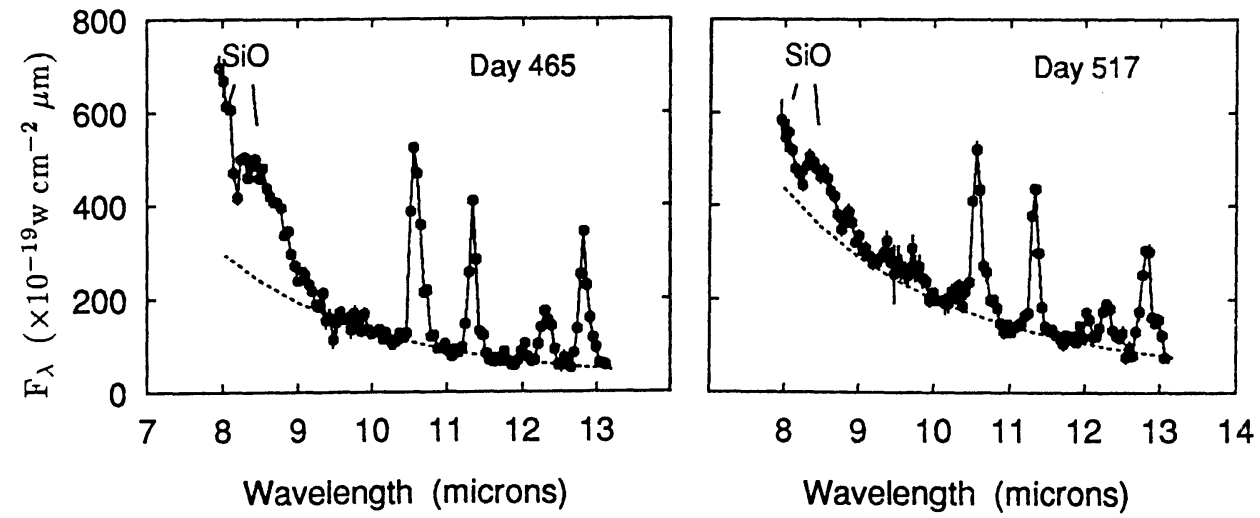

Fig 1. Spectra at 8-13 $\mu \mathrm{m}$ of SN 1987A 465 and 517 days after the explosion. 


\section{Modelling the SiO emission}

- The emission from the supernova is attributed to the fundamental $\Delta v=1 \mathrm{SiO}$ vibrationrotation band at $8.1 \mu \mathrm{m}$.

- The emission from each rotational component of the vibrational level is calculated and then summed to give the emission from the band at the adopted temperature.

- Fig 2 shows the continuum-subtracted SiO emission on Day 465 together with the 1500 and $2000 \mathrm{~K} \mathrm{SiO}$ emission spectra convolved to the $0.09 \mu \mathrm{m}$ resolution of the UCL spectrometer, (similar fits are also availablefor day 517). Temperatures of 1000 and $2500 \mathrm{~K}$ produce emission bands that are respectively narrower and broader than the observed spectra.

\section{Mass of SiO}

- The mass of $\mathrm{SiO}$ is estimated from the observed intensity of the emission band and the model fits. With a temperature of $1500 \mathrm{~K}$, we obtain a mass of $\mathrm{SiO}$ of $5.610^{-6} \mathrm{M}_{\odot}$ on day 465 and $2.810^{-6} \mathrm{M}_{\odot}$ on day 517 ; a temperature of $2000 \mathrm{~K}$ would decrease the mass by 20 percent while a temperature of $1000 \mathrm{~K}$ would increase it by 50 percent.

- It appears that the mass of SiO decreased between days 465 and 517 and by day 578 the $\mathrm{SiO}$ emission was no longer detectable. This coincides with the onset of emission from dust from the supernova, which started near day 450 and increased beyond day 578 .

- The SiO molecules may be the precursors of silicate grains, in which case the mass of $\mathrm{SiO}$, at about 10 percent of the mass of dust, is close to that required if the $\mathrm{SiO}$ provides the seed for dust condensation to occur, while the excitation temperature is close to the condensation temperature of silicate materials.

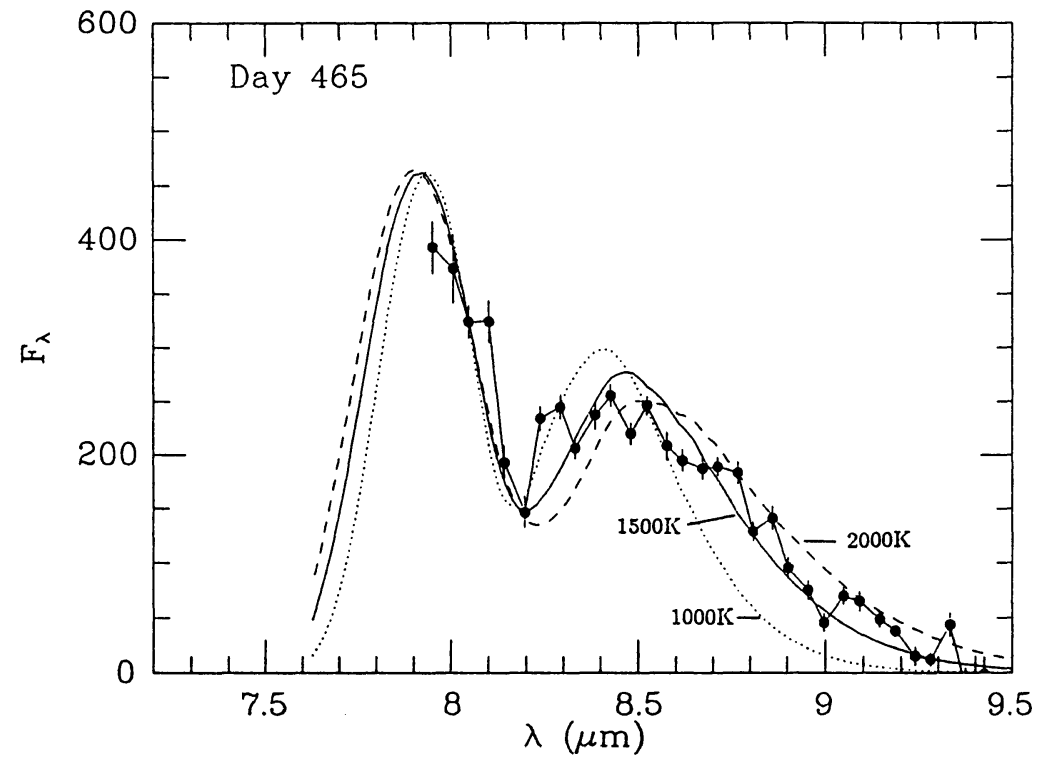

Fig 2. Comparison of observed SiO emission bands with model fits for day 465 . 